\title{
ICSI outcome in surgically retrieved sperm compared with ejaculated sperm control
}

\author{
Pallavi S. Vishwekar ${ }^{1 *}$, Nikita Lad ${ }^{2}$, Mamta Shivtare², Pradnya Shetty ${ }^{2}$
}

\begin{abstract}
${ }^{1}$ Department of Obstetrics and Gynecology, ${ }^{2}$ Department of IVF, DY Patil Medical College and Hospital, Navi Mumbai, Maharashtra, India
\end{abstract}

Received: 03 February 2019

Accepted: 15 February 2019

\section{*Correspondence:}

Dr. Pallavi S. Vishwekar,

E-mail: drpallavibasapure@gmail.com

Copyright: () the author(s), publisher and licensee Medip Academy. This is an open-access article distributed under the terms of the Creative Commons Attribution Non-Commercial License, which permits unrestricted non-commercial use, distribution, and reproduction in any medium, provided the original work is properly cited.

\section{ABSTRACT}

Background: Globally, the prevalence of infertility is around $10 \%$ of the total population. $30 \%$ of these have male factor infertility. Azoospermia is found in $1 \%$ of men, in $20 \%$ of which, the etiology is a bilateral obstruction of the male genital tract while others have non obstructive azoospermia. In azoospermic men sperms are microsurgically retrieved from epididymis and testes by TESA and PESA respectively. The aim of this study was to evaluate the outcomes of intracytoplasmic sperm injection ICSI using surgically retrieved sperm of azoospermic men either obstructive or nonobstructive and to compare it with ejaculated sperms in men having severe oligospermia.

Methods: This was retrospective cohort study conducted based on the data collected from our reproductive endocrinology and infertility unit, 126 ICSI cycles performed during the period of 5 years were taken and divided into two groups, one with patients having ejaculated sperms with oligospermia and other group with patients who had surgically retrieved normal sperms due to azoospermia. Outcome of these ICSI cycles included fertilization, cleavage, biochemical and clinical pregnancy was assessed.

Results: In present study it was found that ICSI outcome was comparable in both the groups with ejaculated sperm and surgically retrieved sperm as fertilization rate (72\% vs $65 \%$ ), Implantation Rate (58 vs 51\%), clinical pregnancy rate (CPR) $(51 \%$ vs $44.82 \%)$ observed with ejaculated or retrieved sperm group respectively showed no statistical difference.

Conclusions: Present study shows that minimally invasive techniques of PESA and TESA can be successfully performed to retrieve sperm for ICSI in the treatment of azoospermic men which gives them the chance to father their biological child. The result of this study indicates that treatment outcomes of PESA/TESA-ICSI cycles compare favourably with that of ICSI using ejaculated sperm.

Keywords: Azoospermia, Ejaculated sperm, ICSI, Infertility, Surgically retrieved sperm

\section{INTRODUCTION}

Inability to conceive after 12 months of regular, unprotected intercourse defines Infertility. ${ }^{1}$ Globally, the prevalence of infertility is around $10 \%$ of the total population. ${ }^{2}$ The etiology of infertility are ovulatory disorders (25\%), tubal damage (20\%), male factor infertility $(30 \%)$ and uterine or peritoneal factors $(10 \%) .^{3}$ Since long after Steptoe and Edward made contribution with their success of birth of first baby through in vitro fertilization, IVF now has become an effective method for treatment of infertility. ${ }^{4}$ In conventional IVF normal sperm function is essential for fertilization. The advent of intracytoplasmic sperm injection (ICSI) in 1992, which entail the injection of single sperm into the oocyte has 
constituted a breakthrough in the treatment of male factor infertility. ${ }^{5}$ The therapeutic possibilities of ICSI range from cases of male infertility which show poor progressive sperm motility to its application in azoospermic men where sperms are microsurgically retrieved from epididymis and testes.

Spermatozoa can be obtained from testicle by either testicular sperm aspiration (TESA), testicular sperm extraction (TESE)or from epididymis by percutaneous epididymal sperm aspiration (PESA).

Azoospermia is found in $1 \%$ of men, in $20 \%$ of which, the etiology is a bilateral obstruction of the male genital tract. $^{6}$ The most common causes of obstructive azoospermia (OA) include vasectomy and congenital absence of the vas deferens, which is found in 1-2\% of the infertile male population. ${ }^{6}$ The second group of azoospermia men are those with nonobstructive causes (NOA). Patients of this group often have small testis size and elevated follicle stimulation hormone (FSH) levels, which could be characterized as having testicular failure . The common causes of NOA include Klinefelter's syndrome and mumps orchitis. ${ }^{7}$

The outcome of ICSI using non-ejaculated sperm depends on many factors. ${ }^{8}$ These include the etiology of azoospermia, the origin of the retrieved sperm, and the sperm status being fresh or cryopreserved. ${ }^{9}$ The use of thawing cryopreserved sperm could be advantageous over the use of freshly retrieved sperm. One reason for this is the avoidance of hormonal stimulation of the female partner and the risk of having failed sperm retrieved, as it accounts for $50 \%$ of male patients suffering from NOA. ${ }^{10}$

Moreover repeated testicular surgery in subsequent ICSI cycles may cause testicular devascularization and possibly permanent injury. In order to overcome these problems, diagnostic biopsies can be performed and cryopreservation of the spermatozoa can be performed.

The aim of this study was to evaluate the outcomes of intracytoplasmic sperm injection ICSI using surgically retrieved sperm of azoospermic men either obstructive or nonobstructive and to compare it with ejaculated sperms in men having severe oligospermia.

\section{METHODS}

A retrospective cohort study was conducted based on the data collected from present reproductive endocrinology and infertility unit over a period of 5 years. 126 ICSI cycles performed during the period of 5 years were taken and divided into two groups, one with patients having ejaculated sperms with oligospermia and other group with patients who had surgically retrieved normal sperms due to azoospermia. Outcome of these ICSI cycles included fertilization, cleavage, biochemical and clinical pregnancy was assessed.

\section{Inclusion criteria}

- Normoovulatory female partner (with normal hormonal profile)

- Males with mild, moderate, severe oligospermia

- Azoopermic males (obstructive or non obstructive) with normal sperms retrieved after surgical procedure.

\section{Exclusion criteria}

- Patients who cannot meet the above criteria were excluded.

All men who had azoospermia on at least 2 semen analyses were further evaluated. The patients had a comprehensive history taken followed by physical examination including inguinoscrotal examination. Testicular volume was determined by a Prader orchidometer and the status of the epididymis and the presence or absence of vas deferens was noted. Hormone levels including follicle-stimulating hormone were evaluated in all azoospermic men. Other tests for hormones such as serum levels of testosterone, luteinizing hormone, and prolactin were done as indicated. If there was suspicion of obstructive azoospermia, further evaluation, including transrectal ultrasonography, was performed to rule out ejaculatory duct obstruction. All men with azoospermia were counselled for need of further workup including genetic testing. Data, however, is not included in the present analysis due to its lack of direct bearing on the objectives of the study.

Controlled ovarian stimulation was done after evaluation of the female partner that included day 2 transvaginal sonography for AFC (more than 5-7 was considered normal) and blood hormonal profile for FSH $(<12 \mathrm{mIU} / \mathrm{ml})$, Estradiol E2 $(<75 \mathrm{pg} / \mathrm{ml})$ and progesterone $(<1 \mathrm{ng} / \mathrm{ml})$.

Stimulation protocol was gonadotropins (HMG) with antagonist started when the dominant follicle reached $>14 \mathrm{~mm}$ in mean diameter (ciscure $0.25 \mathrm{mgsc}$ ) daily till the day of human chorionic gonadotropin (hcg) or decapeptyl (GnRH agonist) trigger. After starting gonadotropin stimulation, the cycle was monitored with transvaginal sonography. When USG shows 4-5 follicles of 17 to $18 \mathrm{~mm}$, E2 level is more than $500 \mathrm{pg} / \mathrm{ml}$, induction of final oocyte maturation was done by HCG Injection that acts as analogue of LH hormone. After this HCG injection oocyte retrieval was performed after 34 to $36 \mathrm{hrs}$ that is just prior to follicular rupture.

\section{Oocyte retrieval}

The oocytes were retrieved after 34 to 36 hours of HCG trigger, using transvaginal technique by ultrasound guided needle aspiration. 
This procedure was done under short general anesthesia and takes 20 to 25 min depending upon the number of mature follicles. The follicular fluid is aspirated using aspiration needle and immediately sent to laboratory which is besides the OT, for visualization of oocyte cumulus complex under stereomicroscope and is washed with flushing media and then placed in incubation media for incubation for 2 hours at $37 \mathrm{c}$ at $5 \% \mathrm{CO}_{2}$ concentration. The oocytes are then denuded of cumulus cells mechanically and using hyaluronidase enzyme in HEPES buffer media under stereomicroscope and then incubated until the time of ICSI.

\section{Sperm preparation}

In cases with oligospermia, fresh ejaculated semen sample was collected by masturbation on the day of oocyte retrieval and analysed after liquefaction under Makler chamber, using density gradient method most progressively motile sperms were separated from dead sperms and debris.

\section{Method}

$1 \mathrm{ml}$ of $80 \%$ density gradient was gently over layed with $40 \%$ sperm gradient medium in a conical tube using sterile pipette, on top of this $1 \mathrm{ml}$ of semen sample is layered. Without disturbing the layers centrifugation is done at $1500 \mathrm{rpm}$ for $20 \mathrm{~min}$. Discarding the supernatant, the pellet with as little as $80 \%$ solution as possible is left behind. In a new test tube $5 \mathrm{ml}$ media is taken and pellet is transferred to this tube and centrifuged at 1500rpm for 10min. Supernatant containing debris, leucocytes, dead sperms is discarded without disturbing the pellet which is resuspended in $1 \mathrm{ml}$ of sperm wash media. This final solution is incubated at $37 \mathrm{C}$ and $5 \% \mathrm{CO}_{2}$ until ICSI.

In cases with azoospermia percutaneous epididymal sperm aspiration (PESA) was tried, if it failed to recover sperms then testicular sperm aspiration (TESA) was done.

\section{Method}

For PESA, a fine needle (e.g., 26 gauge) attached to a $1 \mathrm{ml}$ tuberculin syringe is inserted through the scrotal skin into the epididymis. Negative pressure is created by pulling the syringe plunger while the tip of the needle is gently moved in and out inside the epididymis until a clear fluid is seen coming into the syringe. The amount of fluid aspirated is often minimal $(\sim 0.1 \mathrm{ml})$, except in cases of CAVD in which $0.3-1.0 \mathrm{ml}$ may be obtained. The aspirate is flushed into a tube containing warm sperm medium. The tube containing the epididymal aspirate is taken to the laboratory for immediate microscopic examination. PESA is repeated at a different site from cauda to caput epididymis until adequate number of motile sperms is retrieved. If PESA fails to retrieve motile sperm, testicular sperm retrieval can be attempted at the same operative time.
Despite minor technical variations, the common principle of all methods described for TESA involves the needle insertion through the scrotal skin into the testis. Then, testicular parenchyma is percutaneously aspirated using fine e.g. 22 gauge or large diameter needle e.g., 18 gauge. The needle is usually inserted at the anteromedial or anterolateral portion of the superior testicular pole, in an oblique angle towards the medium and lower poles. These areas are least likely to contain major branches of the testicular artery running superficially underneath the albuginea. Loupe-magnification may be used to avoid small vessels seen through the skin. Negative pressure is created by pulling the syringe plunger while the tip of the needle is moved in and out the testis in an oblique plane to disrupt the seminiferous tubules and sample different areas. The specimen is flushed into a tube containing warm sperm medium and is immediately transferred to the laboratory for microscopic examination. TESA or TESE may be performed at the contralateral testis if insufficient or no sperm are obtained.

\section{Intracytoplasmic sperm injection technique (ICSI)}

The ICSI procedure is carried out 38-42 hours after HCG injection and is performed only for metaphase II (MII) oocytes that had extruded their first polar bodies. The microinjection procedure was performed on a heated stage at $37^{\circ} \mathrm{C}$ under an inverted microscope at $400 \times$ magnification. A drop of prepared sperm was placed in an ICSI dish and the sperm was aspirated with an injection pipette and transferred to a $4 \mathrm{ml}$ droplet of polyvinylpyrrolidone solution (PVP), used for immobilization of spermatozoa. Active spermatozoa must first be immobilized; this is accomplished by gently tapering the tail, then it is drawn into the injection pipette from its tail. Meanwhile, the oocytes were placed in $5 \mu 1$. droplets of flushing media which was covered by light weight mineral oil. The oocyte was held by a holding pipette with the polar body at twelve or six o'clock position. The injecting pipette containing immobilized spermatozoon is introduced at three o'clock position through the zona pellucida and oolemma, deep into the ooplasm. After injection, the oocytes were rinsed or washed and stored in $25 \mu \mathrm{l}$. of culture medium under oil in a culture dish. They were incubated at $37^{\circ} \mathrm{C}$ in an atmosphere of $5 \%$ of $\mathrm{CO}_{2}$ for 16-18 hours.

\section{Assessment of fertilization, embryo culture, and zygote and embryo grading}

Fertilization was evaluated 16-18 hours after ICSI. Normal fertilization was defined as zygotes with two pronuclei (2PN) after ICSI. After the ICSI procedure, oocytes were cultured and assessed for the presence of pronuclei after 16-18 hours of incubation. On day 3, all transferable embryos were assessed for blastomere number and regularity as well as presence and volume of cytoplasmic fragmentation. After 2 days of culture in medium, blastocyst formation was evaluated. The scoring assessment for blastocysts was based on the expansion 
state of the blastocyst, and on the consistency of the inner cell mass and trophectoderm cells. Grade 1 day 3 embryo morphologies (eight cells, blastomeres of equal size, and no cytoplasmic fragments) or day 5 blastocysts (full blastocysts onward; development of the inner cell mass with numerous, tightly packed cells; and trophectoderm, with many cells forming a cohesive epithelium) were considered as "top-quality" embryos.

\section{Embryo transfer and implantation}

The number of embryos to be transferred depends on the number available. For each couple, one to three embryos were transferred. Embryo transfer was performed on day 3 or 5 of retrieval, with the use of a thin labotech catheter. Support of the luteal phase was achieved by progesterone i.e. natural micronized progesterone intramuscular injection and progesterone vaginal gel $8 \%$ started 3 or 5 days before embryo transfer depending on the stage of embryo cleavage stage or blastocyst.
Biochemical pregnancy was assessed by serum $\beta \mathrm{HCG}$ concentration 15 days and 17days post-embryo transfer ( $\beta \mathrm{HCG}$ higher than $5 \mathrm{mIU} / \mathrm{ml}$ ) and confirmed by the presence of a gestational sac on transvaginal sonography at least four weeks after embryo transfer, confirmed clinical pregnancy.

\section{RESULTS}

In this study 126 ICSI cycles were included of which 97 were ICSI performed from ejaculated sperms in oligospermic men (Group A) and 29 were with ICSI performed with surgically retrieved sperm (Group B). Table 1 shows that authors had studied 126 cases and 180 ICSI cycles, of which 97 cases (137 cycles) were with ICSI performed with ejaculated sperms and 29 cases (43 cycles) were with ICSI performed with surgically retrieved sperms. Both of these groups had almost statistically equal number of retrieved oocytes and metaphase II oocytes as the $\mathrm{p}$ value was $>0.05$.

Table 1: Characteristics of studied cases.

\begin{tabular}{|c|c|c|c|}
\hline & Group A (ejaculated sperms) & Group B (TESA/PESA sperms) & P value \\
\hline Number of cases $(n=126)$ & $97(76.98 \%)$ & $29(23.01 \%)$ & $0.521 *$ \\
\hline Number of cycles $(n=180)$ & $137(76.11 \%)$ & $43(23.88 \%)$ & $0.472 *$ \\
\hline Female age & $30.23 \pm 4.32$ & $29 \pm 5.23$ & $0.248 *$ \\
\hline Male age & $34.8 \pm 2.8$ & $36.37 \pm 1.9$ & $0.842 *$ \\
\hline E2 at HCG administration & $3000 \pm 467$ & $2800 \pm 660$ & $0.079 *$ \\
\hline Number of retrieved oocytes & $9.35 \pm 1.41$ & $9.9 \pm 1.1$ & $0.527 *$ \\
\hline Number of metaphase II oocytes & $8.88 \pm 1.05$ & $9.20 \pm 1.0$ & $0.642 *$ \\
\hline
\end{tabular}

$\mathrm{P}<0.05=$ significant, $\mathrm{P}>0.05=$ not significant, tabulated value $=3.84$, *chi-square test does not show statistical difference between groups, $\mathrm{p}$ value and significance

Table 2: Comparison between two groups in terms of sperm motility and fertilisation.

\begin{tabular}{|c|c|c|c|}
\hline Variables & Ejaculated sperms & TESA/PESA sperms & $\mathbf{P}$ value \\
\hline Motile sperms \%/cycle & $90.33 \pm 3.16$ & $87 \pm 4.15$ & $0.457 *$ \\
\hline Oocyte maturation MII retrieved oocytes & $582(75 \%)$ & $170(73 \%)$ & $0.845^{*}$ \\
\hline Fertilization rate & $419(72 \%)$ & $110(65 \%)$ & $0.941 *$ \\
\hline Cleavage rate & $335(80 \%)$ & $86(79 \%)$ & $0.487 *$ \\
\hline Biochemical pregnancy cases & $56(58 \%)$ & $15(51 \%)$ & $0.527 *$ \\
\hline Clinical pregnancy cases & $49(51 \%)$ & $13(44.82 \%)$ & $0.574 *$ \\
\hline
\end{tabular}

$\mathrm{P}<0.05=$ significant, $\mathrm{P}>0.05=$ not significant, tabulated value $=1.96$, *Z-test does not show statistical difference between groups, $\mathrm{p}$ value and significance

The total no of oocytes of M II maturity in ejaculated sperm group were $582(75 \%)$ and in surgically retrieved sperm group was 170 (73\%). Of these $72 \%$ and $65 \%$ showed signs of fertilization in these group respectively. Out of these fertilized oocytes $80 \%$ and $79 \%$ showed growth to cleavage stage respectively. The biochemical and clinical pregnancy were seen in $58 \%$ and $51 \%$ in ICSI with ejaculated sperm group while the same were $51 \%$ and $44.82 \%$ in the other group in which ICSI was performed using surgically retrieved sperm through
TESA or PESA. The test of significance did not show any statistically significant difference in both the groups (Table 2).

\section{DISCUSSION}

The options when treating patients with azoospermia are the choice between using surgical correction of obstruction, surgically retrieved sperm or resorting to using of donor sperms. Patients are reluctant to use donor 
sperm, as it does not give the patient their genetically own children. Patients with azoospermia where surgical correction is not feasible or has failed, recovery of spermatozoa can be made from either the epididymis or the testis. Surgical sperm retrieval and ICSI provided the opportunity for men with azoospermia to father biological children

The approach to azoospermic patients has changed significantly with the introduction of sperm retrieval techniques and assisted reproduction, especially the ICSI. In addition to improving pregnancy rates using sperm from ejaculated semen, ICSI has opened new possibilities for achieving pregnancy with sperm retrieved from the epididymes or testes which have been performed for more than 10 years. Satisfactory results have been achieved in various studies using these techniques. ${ }^{11}$

Aboulghar and colleagues and other authors did not find any significant difference between the treatment results following ICSI with ejaculated sperm and with epididymal sperm. ${ }^{12,13}$ A recent meta-analysis of surgical sperm retrieved in azoospermic patients, however, concluded that sperm origin does not affect cycle outcome. ${ }^{14}$

Using ejaculated sperm for ICSI in the treatment of male factor infertility showed that there was no correlation between sperm motility, density, or percentage of abnormal forms and the fertilization rates and pregnancy rates, as long as motile, morphologically well shaped sperm could be found for the microinjection. ${ }^{15}$ The procedure of ICSI with surgically retrieved sperms is very helpful for it prevents repeated sperm retrieval through the microdissection procedure, therefore there is less general anesthesia risk and also less tissue scarring. ${ }^{9}$ Also efficient non-ejaculated sperm preservation allows the independence of the surgical sperm retrieval from controlled ovarian stimulation and oocyte retrieval. ${ }^{16-18}$ Thus, unnecessary ovarian stimulation is prevented in cases where no sperm can be retrieved, and oocyte retrieval planning is possible in the others.

Present data shows that there were no differences in the fertilization rate between the two groups of ejaculated sperms and surgically retrieved sperms in azoospermia while the majority of the retrospective analysis done previously showed there was a lower fertilization rate in nonobstructive azoospermia after performing the ICSI procedure with thawed spermatozoa. This could be explained by the fact that the causes of nonobstructive azoospermia were not researched in this study. A possible reason for the lower fertilization rates after ICSI in azoospermia might be the lower concentration of spermatozoa, so that the possibility of choosing a normal mature spermatozoon is reduced, as previous studies have shown. A possible limitation of this study is that fewer numbers of patients in present center were found with obstructive azoospermia who also had sperm cryopreserved through microdissection surgery. Instead of that, authors found most of them had testicular sperm aspiration under local anesthesia, which explained the fewer number of obstructive azoospermia in the comparison. In addition, the causes of nonobstructive azoospermia were not researched in this study, therefore further analysis is needed in future studies. Although a body of research implies that the freshly ejaculated sperms are better than surgically retrieved sperms for ICSI, authors found almost equal results. Abduljabbar et al, reached to this conclusion that the fertilization rates are not affected by the source of the sperm but the quality of embryos gets better with ejaculated sperm when compared with sperm from TESE which corroborated in a case report on men with cryptozoospermia, however present study showed equally good quality embryos and implantation rates in both groups. ${ }^{19,21}$

Nagy et al and Aboulghar et al reported that the fertilization and pregnancy rates by testicular spermatozoa were significantly lower than those of ejaculated and epididymal spermatozoa in nonobstructive azoospermia. ${ }^{19,20}$ The sperm extracted from testicular and epididymis in present study had similar fertility outcomes as ejaculated sperms.

In accordance to present study results, some researchers have shown that the fertilization rate of testicular extracted spermatozoa from non-obstructive azoospermic men is comparable to ejaculated spermatozoa in men with unexplained fertility and also stated that the fertilization rate of severe oligospermia or cryptozoospermia is similar to that of testicular spermatozoa source. ${ }^{20,22,23}$

In present study no significant association of fertilization rate $(72 \%$ vs $65 \%)$, implantation rate $(58 \%$ vs $51 \%)$, clinical pregnancy rate (CPR) (51\% vs $44.82 \%)$ was observed with ejaculated or retrieved sperm group respectively. Mohammad-Ali Khalili et al analyzed that the fertilization rate for the groups whose sperms had been obtained by ejaculation, PESA, and TESE was $72.1 \%, 73.6 \%$, and $51.3 \%$, respectively. ${ }^{24}$ Naru $\mathrm{T}$ et al carried out a study on 517 couples and found no significant differences were seen in pregnancy and miscarriage rates with surgically retrieved and ejaculated sperm. ${ }^{25}$ Amrijannati et al performed nineteen ICSI cycles performed with testicular spermatozoa and the rest of cycles $(n=208)$ carried out with ejaculated spermatozoa. $^{26}$ Result analysis showed similar fertilization rate between testicular and ejaculated spermatozoa (respectively, 60\% versus 68\%, $\mathrm{P} \geq 0.05$ ). Jamal $\mathrm{W}$ et al found that there were no significant differences in fertilization rate or cleavage rate between the ejaculated and the surgically retrieved groups. ${ }^{27}$ Goker EN et al concluded that the fertilizing ability of sperm in ICSI is highest with ejaculated sperm and lowest with sperm extracted by testicular biopsy. ${ }^{28}$ Also, the clinical PRs are significantly lower in ICSI with sperm from testicular biopsy. However, the outcomes of pregnancies are not affected by using surgically retrieved sperm from ejaculated semen. Ben-Ami I et al found 
there were no significant differences in fertilization rates between the two subgroups. ${ }^{29}$ A comparison between testicular spermextraction (TESE) versus ejaculated sperm cycles revealed significantly higher implantation rate $(20.7 \%$ vs $5.7 \%)$, higher PR (42.5\% vs $15.1 \%)$, and higher take home baby rate $(27.5 \%$ vs $9.4 \%)$. Negri L et al found that in cases of persistent necrozoospermia, testicular sperm should be favoured over ejaculated sperm. $^{30}$ These studies in literature, Jamal W et al, Amrijannati et al, Tahira Naru et al, found no significant difference in ICSI outcomes with testicular and ejaculated, which are consistent with present study results. ${ }^{25-27}$

Present study shows equally good fertility outcome through ICSI in infertile couples with azoospermic male partners with use of surgical sperm retrieval techniques.

The limitation of present study was the sample size.

\section{CONCLUSION}

Present study shows that minimally invasive techniques of PESA and TESA can be successfully performed to retrieve sperm for ICSI in the treatment of azoospermic men which gives them the chance to father their biological child. The result of this study indicates that treatment outcomes of PESA/TESA-ICSI cycles compare favourably with that of ICSI using ejaculated sperm.

Funding: No funding sources Conflict of interest: None declared

Ethical approval: Not required

\section{REFERENCES}

1. Edmund Sabanegh Jr, Ashok Agarwal, Marc Goldstein Male Infertility, Alan J. Wein, Campbellwalsh urology. $10^{\text {th }}$ ed. Saunders Elsevier: Philadelphia, USA; 2012:616-648.

2. Hull MG, Glazener CM, Kelly NJ, Conway DI, Foster PA, Hinton RA, et al. Population study of causes, treatment, and outcome of infertility. Br Med J (Clin Res Ed). 1985;291(6510):1693-7.

3. NICE: National institute for health and clinical excellence. Fertility assessment and treatment for people with fertility problems. NICE clinical guidelines2013;156. Available at: www.nice.org.UK/guidance/c9156.

4. Steptoe PC, Edwards RG. Birth after the reimplantation of a human embryo. Lancet. 1978;312(8085):366.

5. Palermo G, Joris H, Devroey P, Van Steirteghem AC. Pregnancies after intracytoplasmic sperm injection of single spermatozoon into an oocyte. Lancet. 1992;340:17-8.

6. Dohle GR, Ramos L, Pieters MH, Braat DD, Weber RF. Surgical sperm retrieval and intracytoplasmic sperm injection as treatment of obstructive azoospermia. Hum Reprod (Oxford, England). 1998;13(3):620-3.

7. Nicopoullos JD, Gilling-Smith C, Almeida PA, Norman-Taylor J, Grace I, Ramsay JW. Use of surgical sperm retrieval in azoospermic men: a metaanalysis. Fertil Steril. 2004;82(3):691-701.

8. Friedler S, Raziel A, Schachter M, Strassburger D, Bern O, Ron-El R. Outcome of first and repeated testicular sperm extraction and ICSI in patients with non-obstructive azoospermia. Hum Reprod. 2002;17(9):2356-61.

9. Friedler S, Raziel A, Strassburger D, Schachter M, Soffer Y, Ron-El R. Factors influencing the outcome of ICSI in patients with obstructive and nonobstructive azoospermia: a comparative study. Hum Reprod. 2002;17(12):3114-21.

10. Verheyen G, Vernaeve V, Van Landuyt L, Tournaye H, Devroey P, Van Steirteghem A. Should diagnostic testicular sperm retrieval followed by cryopreservation for later ICSI be the procedure of choice for all patients with non-obstructive azoospermia?. Hum Reprod. 2004;19(12):2822-30.

11. Palermo GD, Cohen J, Alikani M, Adler A, Rosenwaks Z. Intracytoplasmic sperm injection: a novel treatment for all forms of male factor infertility. Fertil Steril. 1995;63:1231-40.

12. Meniru GI, Gorgy A, Batha S, Clarke RJ, Podsiadly BT, Craft IL. Studies of percutaneous epididymal sperm aspiration (PESA) and intracytoplasmic sperm injection. Hum Reprod Update. 1998;4:57-71.

13. Aboulghar MA, Mansour RT, Serour GI, Fahmy I, Kamal A, Tawab NA, et al. Fertilization and pregnancy rates after intracytoplasmic sperm injection using ejaculate semen and surgically retrieved sperm. Fertil Steril. 1997;68:108-11.

14. Nicopoullos JD, Gilling-Smith C, Almeida PA, Norman-Taylor J, Grace I, Ramsay JW. Use of surgical sperm retrieval in azoospermic men: a metaanalysis. Fertil Steril. 2004;82:691-701.

15. Mansour RT, Aboulghar MA, Serour GI, Amin Y, Ramzi AM. The effect of sperm parameters on the outcome of intracy-toplasmic sperm injection. Fertil Steril. 1995;64:982-6.

16. Oates RD, Lobel SM, Harris DH, Pang S, Burgess CM, Carson RS. Efficacy of intracytoplasmic sperm injection using intentionally cryopreserved epididymal spermatozoa. Hum Reprod. 1996;11(1):133-8.

17. Nudell DM, Conaghan J, Pedersen RA, Givens CR, Schriock ED, Turek PJ. The mini-micro-epididymal sperm aspiration for sperm retrieval: a study of urological outcomes. Hum Reprod (Oxford, England). 1998;13(5):1260-5.

18. Janzen N, Goldstein M, Schlegel PN, Palermo GD, Rosenwaks Z. Use of electively cryopreserved microsurgically aspirated epididymal sperm with IVF and intracytoplasmic sperm injection for obstructive azoospermia. Fertil Steril. 2000;74(4):696-701. 
19. Koscinski I, Viville S, Lefebvre-Kahlil V, Defossez A, Rigot JM. Pregnancies in cryptozoospermia with sperm ejaculated one day before ICSI: four case reports. J Androl. 2007;28(1):15-20.

20. Bendikson KA, Neri QV, Takeuchi T, Toschi M, Schlegel PN, Rosenwaks Z, et al. The outcome of intracytoplasmic sperm injection using occasional spermatozoa in the ejaculate of men with spermatogenic failure. J Urol. 2008;180(3):1060-4.

21. Nagy Z, Liu J, Cecile J, Silber S, Devroey P, Van Steirteghem A. Using ejaculated, fresh, and frozenthawed epididymal and testicular spermatozoa gives rise to comparable results after intracytoplasmic sperm injection. Fertil Steril. 1995;63(4):808-15.

22. Abhyankar N, Kathrins M, Niederberger C. Use of testicular versus ejaculated sperm for intracytoplasmic sperm injection among men with cryptozoospermia: a meta-analysis. Fertil Steril. 2016;105(6):1469-75.

23. Ubaldi F, Nagy ZP, Rienzi L, Tesarik J, Anniballo R, Franco G, et al. Reproductive capacity of spermatozoa from men with testicular failure. Hum Reprod. 1999;14(11):2796-800.

24. Khalili MA, Manouchehri MA, Dehghani V. Treatment outcome following intracytoplasmic injection of sperm retrieved from ejaculate, epididymis, or testis of infertile men. Arch Iranian Med. 2004;7(3):232-6.

25. Naru T, Sulaiman MN, Kidwai A, Ather MH, Waqar $\mathrm{S}$, Virk S, et al. Intracytoplasmic sperm injection outcome using ejaculated sperm and retrieved sperm in azoospermic men. Urol J. 2008;5(2):106-10.
26. Amirjannati N, Heidari-Vala H, Akhondi MA, Hosseini Jadda SH, Kamali K, Sadeghi MR. Comparison of intracytoplasmic sperm injection outcomes between $\mathrm{n}$ spermatozoa retrieved from testicular biopsy and from ejaculation in cryptozoospermic men. Androl. 2012;44 Suppl 1:704-9.

27. Jamal W, Vélez MP, Zini A, Phillips S, Hemmings R, Kadoch IJ. Surgically retrieved spermatozoa versus ejaculated spermatozoa in modified natural IVF- ICSI cycles. Reprod Biomed Online. 2012;25(3):242-7.

28. Göker EN, Sendag F, Levi R, Sendag H, Tavmergen E. Comparison of the ICSI outcome of ejaculated sperm with normal, abnormal parameters and testicular sperm. Eur J Obstet Gynecol Reprod Biol. 2002;104(2):129-36.

29. Ben-Ami I, Raziel A, Strassburger D, Komarovsky D, Ron-El R, Friedler S. Intracytoplasmic sperm injection outcome of ejaculated versus extracted testicular spermatozoa cryptozoospermic men. Fertil Steril. 2013;99(7):1867-71.

30. Negri L, Patrizio P, Albani E, Morenghi E, Benaglia $\mathrm{R}$, Desgro $\mathrm{M}$, et al. ICSI outcome is significantly better with testicular spermatozoa in patients with necrozoospermia: a retrospective study. Gynecol Endocrinol. 2014;30(1):48-52.

Cite this article as: Vishwekar PS, Lad N, Shivtare M, Shetty P. ICSI outcome in surgically retrieved sperm compared with ejaculated sperm control. Int J Reprod Contracept Obstet Gynecol 2019;8:869-75. 